\title{
Too much of a good thing
}

Culture of primary lymphocytes at physiological oxygen levels better maintains intracellular redox state.

Cells are typically cultured at atmospheric oxygen levels, which is approximately $20 \%$. This is several fold higher than that encountered by cells in the body: $2-5 \%$ oxygen for cells in tissue or a maximum of $12 \%$ oxygen in arterial blood (Fig. 1). Since ex vivo cell culture is almost always an attempt to approximate the in vivo milieu, the question of what effects this discrepancy has on cultured cells remains a relevant one. "We came to this via B cells and Mishell-Dutton cultures," says Leonore Herzenberg, coauthor with Leonard Herzenberg, both at Stanford University, on a recent paper examining the effect of incubator oxygen levels on redox state and proliferative responses in cultured $\mathrm{T}$ cells. "It has been known for a long time that antibody responses in B cells are better at physiological oxygen, and that got us thinking, why does anybody grow any cells at atmospheric oxygen at all?"

The researchers compared $\mathrm{T}$ cells that had been freshly isolated from the body with the same cells cultured for three days either at atmospheric (20\%) or at physiological (5\%) oxygen. Using tandem mass spectrometry to compare the redox state of these cells, they saw that in vitro culture for three days diminished the ratio of reduced to oxidized glutathione in both cases, but that this occurred to a considerably greater extent for cells grown at atmospheric oxygen levels. Further, and as they had previously reported, proliferation of $\mathrm{T}$ cells in response to $\mathrm{CD} 3 / \mathrm{CD} 28$ stimulation was greater in atmospheric oxygen. This result was somewhat paradoxical. "Our initial naive reaction was that proliferation should have been higher at physiological oxygen because the cells would be happier," says Leonore Herzenberg, "but if you think about it, you don't usually want unregulated proliferation in vivo." Indeed, the scientists found that the bioavailability of intracellular nitric oxide (iNO), an immunomodulatory molecule that inhibits cell proliferation, remained significantly higher for T cells grown at physiological oxygen levels. Cultures at atmospheric levels, therefore, are likely to lack the appropriate immunomodulatory function of iNO, and this may be reflected in an unregulated proliferative response. "More is not always better," Kondala Atkuri, first author on the paper, emphasized.

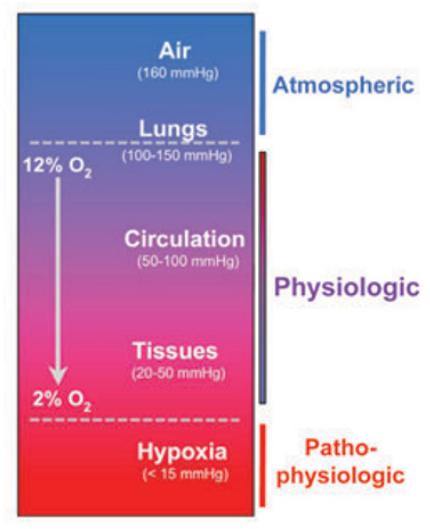

Figure 1 | 0xygen levels encountered by cells. Physiological oxygen ranges from 2 to $12 \%$, whereas cells are cultured at atmospheric levels (20\%). Image courtesy of K. Atkuri and L. Herzenberg.

There is every reason to believe that oxygen levels in culture could affect other primary cells as well. "All mammalian cells live in oxygen," Leonore Herzenberg points out, "so this is widely applicable. But it's likely that it will matter to different degrees for different cell types." Commonly used cell lines, in contrast, have already been adapted over long periods for rapid growth in atmospheric oxygen, suggesting that their redox state is likely to be very dissimilar to that of cells in vivo.

There have been several reports that oxygen levels in culture can affect cell metabolism and function. One reason that the importance of this is not generally appreciated, suggest both Herzenbergs, is that the terminology used to describe incubator oxygen levels is unstandardized. Cells may encounter genuinely hypoxic conditions $(<2 \%)$ in pathophysiological conditions such as the center of tumors. The term hypoxic, however, is sometimes incorrectly used to refer to physiologically relevant $(2-5 \%)$ oxygen levels (Fig. 1), whereas the term normoxic is used for the abnormally high atmospheric (20\%) levels commonly encountered by cells in culture. Standardized and consistent terminology may help to promote a clearer understanding of how to move ex vivo culture conditions for a variety of cell types closer to the in vivo situation.

\section{Natalie de Souza}

\section{RESEARCH PAPERS}

Atkuri, K. et al. Importance of culturing primary lymphocytes at physiological oxygen levels. Proc. Natl. Acad. Sci. USA 104, 4547-4552 (2007). 